\title{
Hormone replacement therapy affects mandibular bone architecture in postmenopausal women: a fractal dimension
} assessment

\author{
Afonso Celso Souza de Assis ${ }^{1}$, Thiago de Oliveira Gamba ${ }^{2}$, Mari Eli Leonelli de Moraes ${ }^{1}$, Luiz César de Moraes ${ }^{1}$, Isadora Luana Flores ${ }^{3 *}$ and \\ Sérgio Lúcio Pereira de Castro Lopes ${ }^{1}$ \\ ${ }^{1}$ Department of Diagnosis and Surgery, São José dos Campos Dental School, UNESP, São José dos Campos, São Paulo, Brazil \\ ${ }^{2}$ Department of Oral Diagnosis, Piracicaba Dental School, UNICAMP, Piracicaba, São Paulo, Brazil \\ ${ }^{3}$ Department of Dentistry, UFJF, Campus Governador Valadares, Governador Valadares, Minas Gerais, Brazil
}

\begin{abstract}
Osteoporosis is an age-related health problem involving estrogen deficiency and the hormone replacement therapy (HRT) is still widely used as an antiresorptive agent. However, the effect on bone microarchitecture needs to be further investigated. To evaluate the effect of HRT on mandibular bone architecture in postmenopausal women using fractal analysis on digital panoramic radiographs. Seventy-five digital panoramic images including 25 images of women aged $20-39$ years (negative control, G1), 25 images of women aged 40-59 years who were not taking HRT (positive control, G2) and 25 images of women aged 40-59 years who were taking HRT (G3) were submitted to fractal analysis using ImageJ software to compare the bone density between the groups. Statistical differences were observed between the fractal indexes in the 3 groups on both sides and the fractal dimension (FD) of group G3 was statistically greater than that of G1 and G2 on both sides. A significant moderate positive correlation $(0.3<\varphi<0.49)$ was found between age and FD on the left mandible in G3. Postmenopausal women under HRT present higher values for FD and these agents can affect the architecture of mandibular bone.
\end{abstract}

\section{Introduction}

Osteoporosis is a disease of the skeletal system characterized by loss of bone mass and clear deterioration of bone microarchitecture resulting in areas highly susceptible to spontaneous fractures $[1,2]$. In postmenopausal women, the combined effects of estrogen deprivation, increased production of follicle-stimulating hormone (FSH) and a decrease in calcium absorption increases the risk of postmenopausal osteoporosis [2,3]. Among the major pharmacological interventions, hormone replacement therapy (HRT) is still widely available for the prevention and treatment of postmenopausal osteoporosis to reduce the rate of bone loss $[2,4]$.

In the jaw bones, an apparent enhancement of the oblique line is relatively common in intraoral and panoramic radiographs, especially in patients aged more than 50 years, because there is an evident loss of trabecular bone mass of the mandible body and low cortical bone loss $[1,2]$ Moreover, resorption of the inferior mandibular cortex can be detected by panoramic radiograph [5]. Thus, as panoramic radiographs are often requested in the dental clinic, this imaging modality can be used as a screening tool to assess the level of bone mineral density and predict the presence of osteoporosis, so that patients can be referred for more complex tests such as bone densitometry $[2,6]$.

Fractal analysis is an effective and useful quantitative method to analyze the trabecular bone pattern through complex measurements of geometric structures throughout the image [7]. Dental radiographs, such as periapical radiographs, provide an efficient method for determining the fractal dimension (FD) by evaluating the changes in bone porosity $[2,6]$. Nevertheless, only seven studies used panoramic radiographs for FD analysis in osteoporotic patients [1,7-12] and no previous articles observed the bone architecture in patients under HRT.

We hypothesize that HRT alters the jaw bone pattern and; therefore, the purpose of the present study was to evaluate the effect of HRT on the architecture of mandibular bone in postmenopausal women using fractal analysis on digital panoramic radiographs.

\section{Materials and methods}

\section{Patients}

This study followed the guidelines of the Declaration of Helsinki and Tokyo for research in humans and was approved by the local ethics committee of São José dos Campos Dental School - UNESP (protocol number: 027/2011-PH/CEP). All patients received verbal information and informed consent was obtained from all participants included in the study. Digital panoramic radiographs of 75 women were distributed into 3 groups as follows: G1, 25 images of women aged 20-39 years (negative control); G2, 25 images of women aged 40-59 years who were

Correspondence to: Dr. Isadora Luana Flores, Área de Estomatologia e Patologia Oral Departamento de Odontologia, Universidade Federal de Juiz de Fora Campus Governador Valadares - UFJF/GV Rua Israel Pinheiro, 2000 Bloco D9 CEP 35020-220 Governador Valadares - MG - Brazil, Tel: +55 3333011000 Ramal 6539, E-mail: isadoraluanaflores@gmail.com

Key words: fractal, hormone replacement therapy, mandible, osteoporosis, panoramic radiography

Received: January 09, 2017; Accepted: February 10, 2017; Published: February 13,2017 
not taking HRT with osteoporosis diagnosis (positive control); and G3, 25 images of women aged 40-59 years who were taking HRT with osteoporosis diagnosis. All images of patients were obtained with the assistance of the oral radiology clinic at São José dos Campos Dental School - UNESP and clinical data were collected retrospectively from the patients' charts.

\section{Image collection}

All radiographs were obtained using a digital panoramic unit (ORTHOPHOS XG 5; Sirona, Bemsheim, Germany) operating at 64 $\mathrm{kVp}, 8 \mathrm{~mA}$ and $14.1 \mathrm{~s}$ exposure time. An expert radiologist with 5 years of technical experience performed all radiographic evaluation. Distorted images were excluded.

\section{Trabecular bone analysis}

Morphological analysis was performed to study the trabecular bone pattern according to White et al. [13] Digital radiographs were saved in JPEG format (Joint Photographic Experts Group) with a spatial resolution of 300 pixels and exported to Adobe Photoshop CS5 software (Adobe Systems, San Jose, CA) to selection the region of interest (ROI). Image J software (http://rsb.info.nih.gov/ij/) was used for the FD analysis and comparison of bone texture among groups. The digital images were converted from 8 bits to 16 bits and a ROI measuring $60 \times 120$ pixels was created between the lower premolars. The digital images were segmented to binary images as described by White and Rudolph [14] and the ROI was duplicated and obscured with a Gaussian filter of 35 pixels in diameter according to Yassar et al. [9] Figure 1 shows the sequence of procedures for fractal analysis of the images. Figure 2 illustrates the position of Gaussian Blurred image overlapped the skeletonized image of the same region showing the correct selection of components encompassed ROI.

\section{Statistical analysis}

The Mann-Whitney test was performed to evaluate the differences in the FD values between the right and left sides in each group. The Kruskal-Wallis test followed by post hoc paired comparison was conducted to compare the FD values on each side (right/left) as well as age in each group. The Spearman correlation test was used to analyze the correlation between age and FD on each side in each group. A $P$ value less than 0.05 was considered significant for all statistical tests.

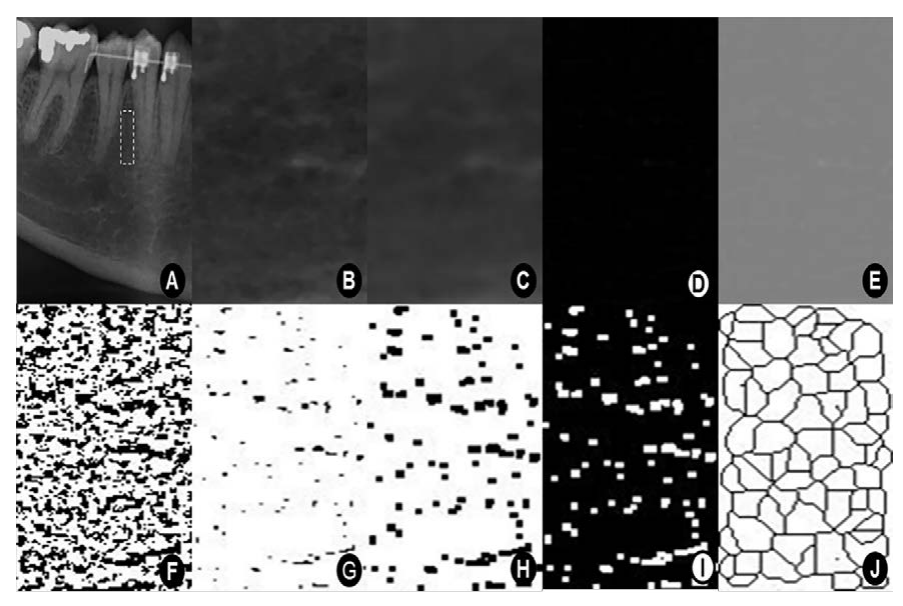

Figure 1. Sequence of fractal analysis of the inferior premolar region. (A) The ROI (60 $\times 120$ pixels) on a panoramic radiograph. (B) The raw image before processing. (C) A Gaussian blurred image. (D) A subtraction image. (E) Added image. (F) A binary image. (G) An eroded image. (H) A dilated image. (I) An inverted image. (J) A skeletonized image.
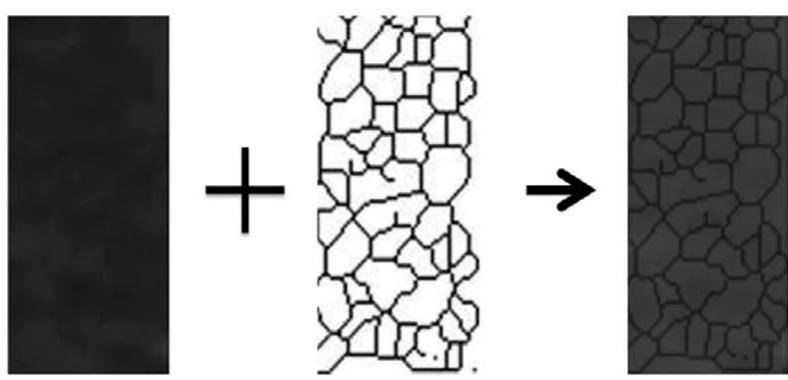

Gaussian Blurred Image

Skeletonized Image

Overlapped Image

Figure 2. Overlapped image including the original or Gaussian blurred image with the skeletonized image. Note the encompassment of bony trabeculation supporting the correct process of ROI selection.

Table 1. Age characteristics of the patients and comparison of ages between groups using the Kruskal-Wallis test followed by post hoc comparison.

\begin{tabular}{|l|c|c|c|}
\hline & G1 & G2 & G3 \\
\hline Sample $(n)$ & 25 & 25 & 25 \\
\hline Minimum & 20.00 & 40.00 & 40.00 \\
\hline Average \pm SD & $24.84 \pm 4.10$ & $46.56 \pm 5.06$ & $50.48 \pm 5.92$ \\
\hline Maximum & 34.00 & 57.00 & 58.00 \\
\hline$P$ value $(\mathrm{G} 1=\mathrm{G} 2=\mathrm{G} 3)$ & & $0.0000^{* *}$ & \\
\hline Post hoc & A & B & C \\
\hline
\end{tabular}

$n$, number; SD, standard deviation. $* *$ Statistically significant difference.

\section{Results}

The characteristics of the patients are showed in Table 1 . The average age of the patients in the negative control group (G1) and the positive control group (G2) was 24.84 and 46.56 years, respectively. The average age of the group under HRT (G3) was 50.48 years. Statistically significant differences were observed between the ages of the 3 groups (Table 1). No statistically significant differences were found between the FD values for the right and left sides in each group (Table 2).

Statistically significant differences were observed in the FD between the groups on both sides. Moreover, the FD in G3 was statistically greater than in G1 and G2 on both sides (Table 3). A moderate positive correlation $(0.3<\phi<0.49)$ was found between age and FD on the left side of the mandible in G3 and the right side of the mandible in G2 group. However, only the first correlation was statistically significant. The other correlations between age and FD were low and showed no statistical significance $(-0.29<\phi<0.29)$. These results are showed in Table 4 .

\section{Discussion}

Panoramic radiographs are regularly requested as a complementary diagnostic examination by dentists and, sometimes, important manifestations of systemic diseases can be found in the gnathic bones [15-19]. Among these, low bone mineral density and morphological changes in trabecular bone reinforce the use of panoramic radiograph together with some clinical parameters to identify patients with osteoporosis [1,20,21]. Although HRT is prescribed as an antiresorptive drug for the treatment of postmenopausal osteoporosis, the effect on the microarchitecture of trabecular bone has been investigated only on calcaneus radiographs showing that HRT interferes with the texture of trabecular bone [22]. The present study provides interesting results by applying a similar methodology to analyze trabecular mandible images 
Table 2. Fractal dimensions (FD) of the right and left sides in each group and $P$ values using the Mann Whitney test.

\begin{tabular}{|l|c|c|c|c|c|c|}
\hline & \multicolumn{2}{|c|}{ G1 } & \multicolumn{2}{c|}{ G2 } & \multicolumn{2}{c|}{ G3 } \\
\hline & RS & LS & RS & LS & RS & LS \\
\hline Patients $(n)$ & 25 & 25 & 25 & 25 & 25 & 25 \\
\hline Minimum & 1.5447 & 1.5138 & 1.5445 & 1.3980 & 1.6507 & 1.6731 \\
\hline Average & 1.6675 & 1.6730 & 1.6735 & 1.6742 & 1.7052 & 1.7074 \\
\hline SD & 0.0465 & 0.0443 & 0.0398 & 0.0635 & 0.0206 & 0.0171 \\
\hline Maximum & 1.7441 & 1.7379 & 1.7192 & 1.7184 & 1.7462 & 1.7373 \\
\hline$P$ value (RS $=$ LS) & \multicolumn{3}{|c|}{0.823} & \multicolumn{2}{|c|}{0.491} & \multicolumn{2}{c|}{0.816} \\
\hline
\end{tabular}

RS: right side; LS: left side; $n$ : number; SD: standard deviation.

Table 3. Comparison of the FD of the right and left sides between the groups using the Kruskal-Wallis test followed by post hoc comparison.

\begin{tabular}{|l|c|c|c|c|c|c|}
\hline & \multicolumn{3}{|c|}{ Right side } & \multicolumn{3}{c|}{ Left side } \\
\hline & G1 & G2 & G3 & G1 & G2 & G3 \\
\hline Average & 1.6675 & 1.6735 & 1.7052 & 1.6730 & 1.6742 & 1.7074 \\
\hline$P$ value $(\mathrm{G} 1=\mathrm{G} 2=\mathrm{G} 3)$ & \multicolumn{3}{|c|}{$0.0003^{* *}$} & \multicolumn{3}{c|}{$0.0002^{* *}$} \\
\hline Post hoc & A & A & B & A & A & B \\
\hline
\end{tabular}

**Statistically significant difference.

Table 4. Spearman coefficients $(\varphi)$ and $P$ values for the correlation between age and FD on each side of the mandible in each group.

\begin{tabular}{|l|c|c|c|c|c|c|}
\hline & \multicolumn{2}{|c|}{ G1 } & \multicolumn{2}{c|}{ G2 } & \multicolumn{2}{c|}{ G3 } \\
\hline & RS & LS & RS & LS & RS & LS \\
\hline$\phi$ & 0.2340 & 0.2030 & 0.3350 & 0.2450 & -0.1220 & 0.4070 \\
\hline$P$ value & 0.2610 & 0.3310 & 0.1020 & 0.2390 & 0.5620 & 0.0433 \\
\hline
\end{tabular}

RS: right side; LS: left side. Moderate statistical significance of Spearman coefficient $(0.3$ $<\varphi<0.49$ ).

on panoramic radiographs of postmenopausal women.

Our results indicate higher values in patients under HRT when the FD of each side was compared between groups. Nevertheless, no statistically significant differences were found between the FD of the right and left sides in the same group of patients. These findings suggest that trabecular bone modifications can occur simultaneously in the same arch and reinforces that HRT interferes with the microarchitecture of the mandible in postmenopausal women as was found also in calcaneus bone [22]. Moreover, a positive correlation between age and FD in the group under HRT and in the group not under HRT (only the former correlation was statistically significant) confirms the possible influence of hormonal therapy on FD values and, consequently, on the architecture of mandible bone. Lespessailles et al. [22] also found a positive correlation between HRT and trabecular bone architecture on calcaneus radiographs.

On the other hand, the results suggest that age is a minor factor with regard to microarchitecture of bone when the FD indexes are compared between women in the same age range with or without HRT. The absence of statistical significance for the positive correlation between age and the negative control group is in agreement with this. Furthermore, FD was significantly lower in a group of postmenopausal women without HRT compared with a group of postmenopausal women with HRT. Although decreased levels of estrogen are considered the primary cause of osteoporosis and the classic HRT are drugs commonly recommended for postmenopausal osteoporosis [2224]. The effect of this treatment modality on the architecture of the jaw bone was not observed in previous studies. Moreover, among the studies using fractal analysis to assess dental panoramic radiographs, there are no evaluations of the architectural pattern of the jaw bone in patients under HRT. The present study has approached this topic with challenging results obtained from an analysis of maxillofacial bone through oral radiographs. Thus, this routine exam in the dental practice can be useful to evaluation of antiresorptive drug effects upon the bone tissue.

Nevertheless, the lack of a group of patients under treatment with other antiresorptive drug classes such as bisphosphonates, calcitonin and, more recently, selective estrogen receptor modulators (SERMs) can be considered as a limitation of this study due to the possibility of comparison of the effect of different agents upon the bone microarchitecture.

\section{Conclusion}

Even so, we conclude that postmenopausal women under HRT present higher FD values than women not under this treatment protocol. Thus, it is highly likely that HRT alters the architecture of mandibular bone, rendering with better bone mineral density. Interestingly, postmenopausal women with osteoporosis taking HRT have better bone mineral density than young healthy women without osteoporosis. This intriguing finding was never reported in the English Literature and we suggest the powerful effect of HRT therapy upon the maxillofacial bone. Nevertheless, only further case-control studies can clarify this assumption. In this context, the oral radiographic examination can be used to screen the bone effects caused by HRT drugs in osteoporotic patients. However, only further studies can elucidate the possible mechanisms involved in the effect of these drugs on bone architecture.

\section{Conflict of interest}

The authors declare no conflict of interest.

\section{Human rights statements and informed consent}

All procedures followed were in accordance with the ethical standards of the responsible committee on human experimentation (institutional and national) and with the Helsinki Declaration of 1975, as revised in 2008. Informed consent was obtained from all patients for being included in the study.

\section{Financial support}

The authors have received no funding for this article.

\section{References}

1. Sindeaux R, Figueiredo PT, de Melo NS, Guimarães AT, Lazarte L, et al. (2014) Fractal dimension and mandibular cortical width in normal and osteoporotic men and women. Maturitas 77: 142-148. [Crossref]

2. Faienza MF1, Ventura A, Marzano F, Cavallo L (2013) Postmenopausal osteoporosis the role of immune system cells. Clin Dev Immunol 2013: 575936. [Crossref]

3. Ramsubeik K, Keuler NS, Davis LA, Hansen KE (2014) Factors associated with calcium absorption in postmenopausal women: a post hoc analysis of dual-isotope studies. J Acad Nutr Diet 114: 761-767. [Crossref]

4. Kanis JA, McCloskey EV, Johansson H, Cooper C, Rizzoli R, et al. (2013) European guidance for the diagnosis and management of osteoporosis in postmenopausal women. Osteoporos Int 24: 23-57. [Crossref]

5. Lee K, Taguchi A, Ishii K, Suei Y, Fujita M, et al. (2005) Visual assessment of the mandibular cortex on panoramic radiographs to identify postmenopausal women with low bone mineral densities. Oral Surg Oral Med Oral Pathol Oral Radiol Endod 100: 226-231. [Crossref]

6. Verheij JG, Geraets WG, van der Stelt PF, Horner K, Lindh C, et al. (2009) Prediction of osteoporosis with dental radiographs and age. Dentomaxillofac Radiol 38: 431-437. [Crossref] 
7. Koh KJ, Park HN, Kim KA (2012) Prediction of age-related osteoporosis using fractal analysis on panoramic radiographs. Imaging Sci Dent 42: 231-235. [Crossref]

8. Oliveira ML, Pedrosa EF, Cruz AD, Haiter-Neto F, Paula FJ, et al. (2013) Relationship between bone mineral density and trabecular bone pattern in postmenopausal osteoporotic Brazilian women. Clin Oral Investig 17: 1847-1853. [Crossref]

9. Yasar F, Akgünlü F (2005) Fractal dimension and lacunarity analysis of dental radiographs. Dentomaxillofac Radiol 34: 261-267. [Crossref]

10. Roberts MG, Graham J, Devlin H (2013) Image texture in dental panoramic radiographs as a potential biomarker of osteoporosis. IEEE Trans Biomed Eng 60: 2384-2392. [Crossref]

11. Bollen AM, Taguchi A, Hujoel PP, Hollender LG (2001) Fractal dimension on dental radiographs. Dentomaxillofac Radiol 30: 270-275. [Crossref]

12. Law AN, Bollen AM, Chen SK (1996) Detecting osteoporosis using dental radiographs: a comparison of four methods. J Am Dent Assoc 127: 1734-1742. [Crossref]

13. White SC (2002) Oral radiographic predictors of osteoporosis. Dentomaxillofac Radiol 31: 84-92. [Crossref]

14. White SC, Rudolph DJ (1999) Alterations of the trabecular pattern of the jaws in patients with osteoporosis. Oral Surg Oral Med Oral Pathol Oral Radiol Endod 88: 628-635. [Crossref]

15. Friedlander AH, Chang TI, Aghazadehsanai N, Berenji GR, Harada ND, et al (2013) Panoramic images of white and black post-menopausal females evidencing carotidcalcifications are at high risk of comorbid osteopenia of the femoral neck. Dentomaxillofac Radiol 42: 20120195. [Crossref]

16. Ramesh A, Soroushian S, Ganguly R (2013) Coincidence of calcified carotid atheromatous plaque, osteoporosis, and periodontal bone loss in dental panoramic radiographs. Imaging Sci Dent 43: 235-243. [Crossref]

17. Bodade PR, Mody RN (2013) Panoramic radiography for screening postmenopausal osteoporosis in India: a pilot study. Oral Health Dent Manag 12: 65-72. [Crossref]

18. Caputo BV, Traversa-Caputo GC, Costa C, Giovani EM (2013) Evaluation of bone alterations in the jaws of HIV-infected menopausal women. Braz Oral Res 27: 231237. [Crossref]

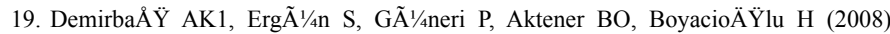
Mandibular bone changes in sickle cell anemia: fractal analysis. Oral Surg Oral Med Oral Pathol Oral Radiol Endod 106: e41-48. [Crossref]

20. Gaur B, Chaudhary A, Wanjari PV, Sunil M, Basavaraj P (2013) Evaluation of panoramic Radiographs as a Screening Tool of Osteoporosis in Post-Menopausal Women: A Cross Sectional Study. J Clin Diagn Res 7: 2051-2055. [Crossref]

21. Ferreira Leite A, de Souza Figueiredo PT, Ramos Barra F, Santos de Melo N, de Paula AP (2011) Relationships between mandibular cortical indexes, bone mineral density, and osteoporotic fractures in Brazilian men over 60 years old. Oral Surg Oral Med Oral Pathol Oral Radiol Endod 112: 648-656. [Crossref]

22. Lespessailles E, Poupon S, Niamane R, Loiseau-Peres S, Derommelaere G, et al (2002) Fractal analysis of trabecular bone texture on calcaneus radiographs: effects of age, time since menopause and hormone replacement therapy. Osteoporos Int 13: 366-372. [Crossref]

23. Gambacciani M1 (2013) Selective estrogen modulators in menopause. Minerva Ginecol 65: 621-630. [Crossref]

24. Maeda SS, Lazaretti-Castro M2 (2014) An overview on the treatment of postmenopausal osteoporosis. Arq Bras Endocrinol Metabol 58: 162-171. [Crossref]

Copyright: $(2017$ Assis ACS. This is an open-access article distributed under the terms of the Creative Commons Attribution License, which permits unrestricted use, distribution, and reproduction in any medium, provided the original author and source are credited. 